\section{'Foveal Tritanopia'}

IN his book on colour vision, Dr. W. D. Wright ${ }^{1}$ discusses blue-blindness of the retinal fovea, which was described by A. Koenig ${ }^{2}$ fifty years ago and is now maintained by Willmer ${ }^{3}$ and himself for the same reasons: "This effect does not appear to have been the subject of further extensive study and although other workers were aware of Koenig's observation the general attitude appears to have been one of scepticism. Indeed most people either ignored or were ignorant of the phenomenon until Willmer recently reawakened interest in the subject." Dr. Wright has apparently overlooked the controversy between E. Hering and A. Koenig.

Koenig's ${ }^{2}$ hypothesis that the visual purple and yellow are the photochemical substances for grey and blue sensations, the rods the colour-receptors, and the cones only dioptric means to throw the retinal images on the rods, led him to the assumption that the purple-free fovea were blue-blind. His observations with colour equations seemed to confirm his assumptions, as in the case of Drs. Willmer and Wright.

Hering ${ }^{4}$ attacked Koenig's observations and conclusions in a paper noteworthy for its concise reasoning and the sharpness of its criticism. Hering showed that direct foveal observation of small blue test objects demonstrates the perfect blue-sensation of the fovea, provided that some precautionary measures are taken which he prescribed with exactness, and that the fovea is the most colour-sensitive spot of the retina.

Conclusions from colour equations and colourmatches on such small retinal fields as the fovea led Koenig to the error of foveal blue-blindness. Colour equations are only valid under three conditions : that the test is of sufficient brightness, that the retinal field has a minimum size, and that the eye is not tired. Hering had shown already in an older paper ${ }^{5}$, before Koenig's publication, that the validity of colour equations is limited and that the latter are not valid on very large fields or very small retinal fields such as the fovea. Colour equations with bluegreen mixtures need much more blue on small retinal fields such as the fovea (1893). Koenig's short reply ${ }^{6}$ was not convincing at all; a more detailed one was promised but never published-a later paper of pathological blue-blindness due to ablatio retinæ and retinitis has nothing to do with the problem in question. Hering ${ }^{7}$ replied and again refuted concisely foveal blue-blindness and Koenig's strange theory of the function of rods and cones, just at the time of the making of the duplicity theory. Everyone of that time interested in the subject knew of Koenig's publication and his lecture at the Academy of Sciences in Berlin, and also of the controversy with Hering. 'There is little doubt that authorities of those days such as v. Kries, Lord Rayleigh and Nagel put such an important question to the test, as was easily done from Hering's description.

Many years after Koenig's death, Sir John Parsons ${ }^{8}$ rejected strongly foveal tritanopia in his standard work on colour vision, saying, "This observation (i.e., Koenig's) is certainly untrue". Curiously, he did not cite Hering on this question, although he quotes him otherwise more than sixty times in his excellent book.

Recently, Prof. H. Hartridge ${ }^{9}$ has rejected foveal tritanopia for precisely the same reasons as E. Hering in the old days, showing in detail that colour equa- tions are not valid on retinal fields so small as the fovea, and direct foveal observation of blue tests gives clear blue response. But there is again no reference to the work of Hering or Helmholtz ${ }^{10}$; the latter was aware of these facts even before Hering. These facts are not at all recent advances, but apparently they have been forgotten nowadays.

1 Gascoyne Road, London, E.9.

${ }^{1}$ Wright, W. D., "Researches on Normal and Defective ColourVision" (1916).

${ }^{2}$ Koenig, A., "Ges. Wissenschaftl. Abhdlg.", 338 (1903).

${ }^{3}$ Willmer, E. N., Nature, 153, 774 (1944). Willmer, E. N., and W right, W. D., Nature, 156, 119 (1945).

'Hering, E., Pflueger's Archiv., 59, 403 (1895).

'Hering, E., Pfiueger's Archiv., 54 (1893). - Koenig, A., Pfueger's Archiv., 60 (1895); “Ges. Wissenschaftl.
Abhdlg.", chapter 25 (1903).

'Hering, E., Pflueger's Archiv., 61 (1895).

- Parsons, J. H., "Introduction to Colour Vision", 81 (1915 and 1924).

- Hartridge, H., Nature, 155, 391 and 657 (1945).

${ }^{10}$ Helmholtz, "Physiol. Optik" (2nd edit., 1896), 374.

ALL workers on colour vision will, I am sure, welcome Dr. Karplus' letter, especially for the historical notes on the dispute between Koenig and Hering. It is quite evident from this that the dichromatism of the central fovea, when reported by Koenig fifty years ago, was the subject of some heated discussion; at the same time, when Willmer ${ }^{1}$ re-discovered the effect in 1944 , it is probably true to say that most workers in the subject had either ignored or were ignorant of both Koenig's results and Hering's discussion of them, and I would have to admit to being among that number. No doubt there were exceptions, and Dr. Karplus is certainly one honourable example; but then I understand that he had the great privilege of working under Koenig in his laboratory - a privilege that may well be unique among those alive to-day.

With regard to the phenomenon itself, I think it should be made clear that the recent observations by Willmer ${ }^{2}$, Thomson ${ }^{3}$ and myself completely substantiate Koenig's original colour-matching experiments. I suspect that many of the difficulties and differences of opinion that have arisen in explaining the dichromatism have had their origin in the different connotations applied to the word 'dichromatism' and also to the misuse of the word 'blind'. As generally understood at the present time, dichromatism implies that colour matching can be carried out by the mixture of only two matching stimuli, and it is in this sense that the central fover has been described as dichromatic, and not in the sense of there being only two qualities of sensation associated with its stimulation. The misuse of the word 'blind' is quite extensive in the frequent description of persons with defective colour vision as being 'colour-blind', although the great majority are in no sense blind to any of the radiations in the visible spectrum, since their essential defect is a reduced ability to discriminate the colour quality of one wave-length from that of another. In the same way, it is incorrect to refer to tritanopia as 'blue-blindness'. The radiations at the short-wave end of the spectrum may be perfectly visible and may even give rise to the sensation of blueness, yet at the same time a tritanope or a tritanopic area of the retina will have a reduced ability to discriminate colours in the blue-green region of the spectrum. It is true that observations by Thomson and myself ${ }^{3}$, and other measurements reported by Dr. W. S. Stiles at Cambridge last July 